\title{
Transposición corregida de los grandes vasos: aspectos clínicos y angiográficos
}

\author{
Dra. Odette Farrú A. ${ }^{1}$; Dr. Ignacio Hernández N. ${ }^{1}$, Dr. Mauricio O'Connell J.1 \\ Dr, Dieter Wolt 2
}

\section{Corrected transposition of the great vessels}

The clinical, hemodynamic and angiographic charactheristics of $\mathbf{4 3}$ cases of corrected transposition of the great vessels (C.T.), are analysed. The average age of patients at diagnosis was 9.6 years, with male predominance (2:1). Thirty eight patients had situs solitus visceralis ( 31 with normocardia, 7 dextroversion) and 5 situs inversus visceralis ( 3 with dextrocardia, 2 with levoversion). Only two cases were not associated with other cardiac defect (one had concomitant Wolff-Parkinson-White syndrome). Ventricular septal defect (VSD) was the most frequent associated cardiac anomaly $(91 \%)$, it occurred in isolated form $(21 \%)$ or in combination with pulmonic stenosis (42\%), left atrioventricular valve defect $(16 \%)$ or both $(12 \%)$. VSD with pulmonic stenosis was the most frequent combination $(42 \%)$, followed by abnormal left atriventricular valve $(30 \%)$, isolated or combined. The electrocardiograms showed a high frecuency of inverted electrical activity: $Q$ waves in right precordial leads and absent $Q$ waves in the left ones in $70 \%$ of the cases and different degrees of $\mathrm{A}-\mathrm{V}$ heart block in $12 \%$ of the patients. Roentgenographically, the most striking feature is an abnormal left heart border that is formed by only two segments (superior-aorta; inferio-systemic ventricle). Hemodynamically two features stand out: difficulty in entcring the pulmonary artery and abnormal course of the cathefer while entering the great vessels (medial and posterior putmonary artery and left anterior aorta). Selective angiocard iography establishes the diagnosis of $C T$ and the associa ted cardiac abnormalities. The effect of the ventricular jnversion on the specialized conduction system, their consequences on the production of rhythen disturbances and on poor results of surgery are analysed.

(Key words: Great vessels, transposition, corrected, situs, associated cardiovasculat abnormalities).

La transposición corregida de los grandes vasos.(TC) representa alrededor de 1 a $2 \%$ de las cardiopatías congénitas y generalmente está asociada a otras anomalias cardíacas que son las que determinan las manifestaciones, el pronóstico, y la decisión del manejo terapéutico (médico o quirúrgico).

Las características anatómicas de las TC están dadas básicamente por una transposición de los grandes vasos y una inversión ventricular. Desde el punto de vista hemodinámico, la sangre venosa sistémica que llega a la aurícula derecha pasa a través de la válvula mitral a un ventículo con morfología de ventriculo izquierdo, del cual sale la arteria pulmonar por detrás y a la derecha de la aorta. La sangre arterial proveniente de los pulmones llega normalmente por las venas pulmonares a la aurícula izquierda pasa por la válvula tricúspide a un ventrículo con morfología de ventrículo derecho, del cual sale la aorta, por

1. Unidad de Cardiología. Departamento de Pediatría. Hospital Roberto del Río.

2. Univesitäts-Kimderklinik (Cardiologische Abteillung). Heidelberg. Alemania. delante y a la izquierda de la arteria pulmonar. En consecuencia, en ausencia de malformaciones asocjadas, no hay trastornos hemodinámicos, ya que la sangre venosa sistémica llega normalmente a los pulmones y la sangre oxigenada de venas pulmonares alcanza la aorta.

Por ser la TC una cardiopatía muy poco frecuente, hemos crejdo de interés presentar los hallazgos clínicos, electrocardiográficos, radiológicos. hemodinámicos y angingráficos de un número relativamente grande de pacientes, que foman parte de un estudio cooperativo con el hospital cardiológico-pediátrico de la Universidad de Heidelberg. Alemania.

\section{MATERIAL Y METODO}

Sc cfectúa un análisis retrospectivo de 43 casos de TC. comprobadas con estudjo clínico, electrocatdiográ. fico, radiológico, hemodinámico y angiográfico selectivo. Dos casos tuvieron comprobación necrópsica, 10 quirúrgísa (correctora total, con 3 fallecidos).

\section{RESULTADOS}

La edad promedio de Ios pacientes fluctuó entre 1 y 28 años, con un promedio de 9,5 afios. 
Predominaron los varones en razón de $2: 1$.

De los 43 pacientes estudiados (Figura 1), 38 presentaron situs solitus visceralis. De éstos, 31 tenían apex cardiaco hacia la izquierda (levocardia o normocardia) y 7 con punta hacia la derecha (dextroversión). Los otros 5 casos tenian situs inversus visceraljs, 3 de ellos con apex a derecha (dextrocardia) y 2 a izquierda (levoversión). En todos los casos se constató concordancia viscero-atrial (el higado al mismo lado que la aurícula derecha).

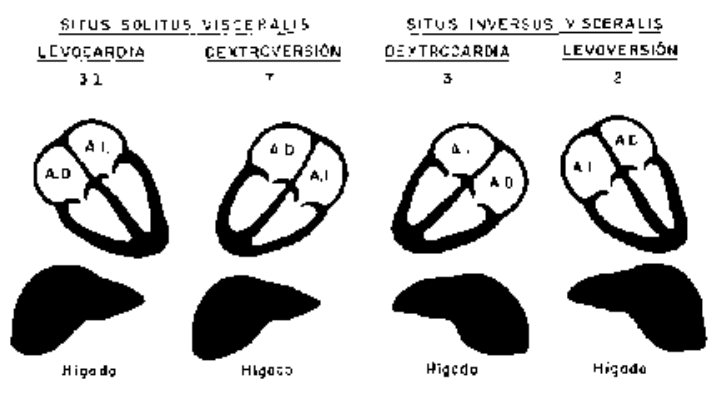

Fig. 1: Distribución de 43 pacientes con transposición corregida, según situs visceralis $y$ cordis.

Sólo se encontraron 2 casos de TC aislada, ‘sin malformaciones asociadas. Uno de ellos presertaba, sin embargo, sindrome de Wolff Parkinson White con crisis de taquicardia paroxisticas reiteradas.

La malformación cardiaca asociada más frecuente fue la comunicación interventricular (ClV), aislada ( $=9 ; 21 \%$ ), asociada a estenosis pulmonar $(n=18 ; 42 \%)$, a malformación de la válvula atrioventricular izquierda $(n=7 ; 16 \%), 0$ a estenosis pulmonar y malformaçión de la válvula atrioventricular izquierda $(\mathbf{n}=5 ; 12 \%)$. 0 sea, la presencia de CIV se comprobó en 39 casos
(91\%). En los 9 casos con CIV aislada, esta era hemodinámicamente significativa en todos (Qp:Qs mayor 2:1). La combinación de CIV $y$ estenosis pulmonar es la asociación más frecuente $(42 \%)$.

La malformación de la válvula atrioventricular izquierda (tricúspide) aislada $(\mathrm{n}=1 ; 2 \%)$ comb . nada con CIV (16\%) o con CIV y estenosis pulmonar (12\%) se presentó en 30\% de los casos. Estenosis pulmonar aislada se presentó sólo en un caso (2\%).

Los trastornos electrocardiográficos más frecuentes fueron: bloqueo auriculoventricular en 6 casos $(14 \%)$, de primer grado en 3 , de segundo grado en 1 y de tercer grado en 2 pacientes. Ondas $Q$ en las derivaciones precordiales derechas y ausentes en la izquierda se observaron en 30 casos $(70 \%)$, aun en presencia de diversas asociaciones de cardiopatías. Ondas $Q$ en precordiales derechas e izquierdas, en 6 casos $(14 \%)$; ondas $T$ positivas en todas las precordiales en 26 casos $(60 \%)$.

La radiografía de tórax simple reveló 35 càsos ( $82 \%$ ) contorno anomal del borde i $\iota q u i e r d o$ do la silueta cardíaca, formado solamente por 2 arcos, el superior, largo y convexo o rectilineo dado por la aorta ascendente (Fig. 2), el inferior. por el ventrículo sistémico (con morfología de derecho). El tamaño cardíaco y el estado de la circulación pulmonar variaron dependicndo de las anomalías asociadas, al igual que Jos hallazgos fonocardiográficos, sin elementos que caracterizarán a la $\mathrm{TC}$.

En el estudio hemodinámico destaca la dificultad para entrar a la arteria pulmonar y el trayecto peculjar de la sonda al cateterizar los grandés vasos: arteria pulmonar medial y posterior, aorta a la isquerda y anterior.
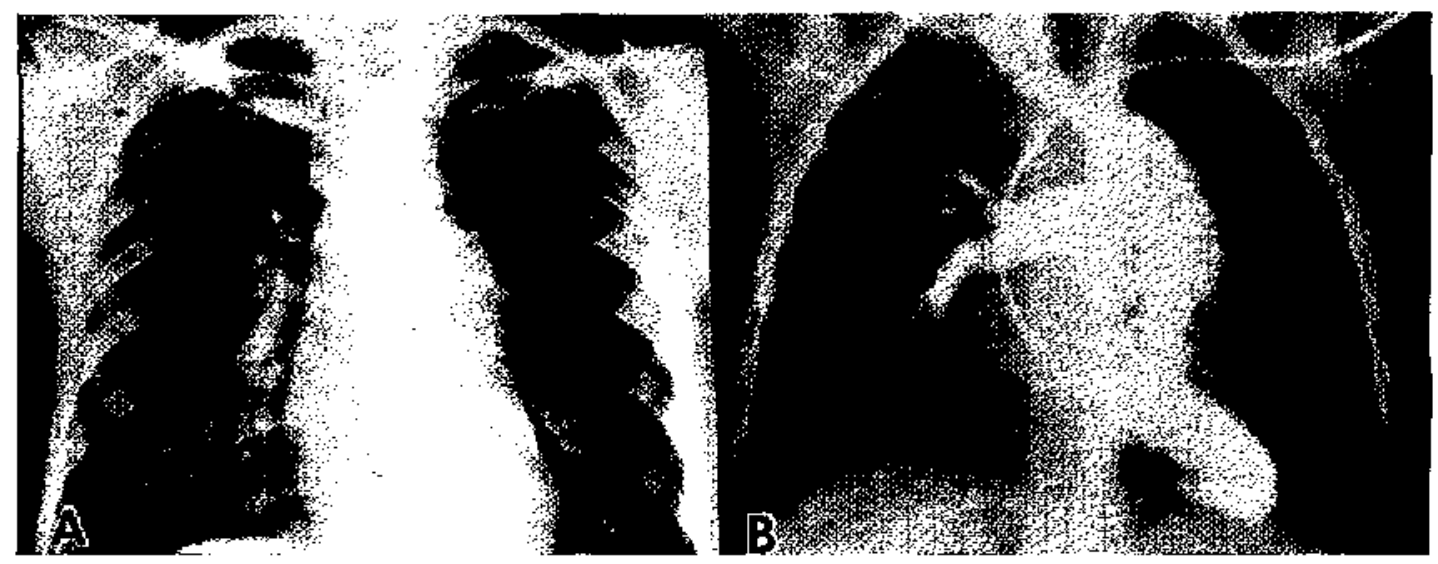

Fig. 2: A) Radiografia simple de lórax. frontal en un faso de trasposición corregida de los grandes yasos: el borde izquicrdo de la silueta cardiaca esta formado por 2 arcos: el superior, largo y algu convexo dado por la aorta ascendente y el inferior por el vientriculo sistémico. B) Angiocardiografía slectiva cn ventrículo venoso. Obsérvese la morfología izquierda de este ventrículo (paredes lisas, no tuberculadas) del cual nace la arteria pulmo nar medial:a través de una CIV se contrasta la aorts a la izquierda. 
La angiocardiografía selectiva (Fig. 3 y 4) permite establecer el diagnóstico y precisar las anomalias asociadas. Deben distinguirse cada vez las 4 posibilidades antes señaladas de acuerdo al situs cordis (Fig. 1). El signo de la "bayoneta" (arteria pulmonar saliendo del ventrículo medial hacia arriba y luego hacia atrás (Fig. 4) se encontró en 18 casos ( $44 \%)$, siendo dudoso en 5 ( $12 \%)$.

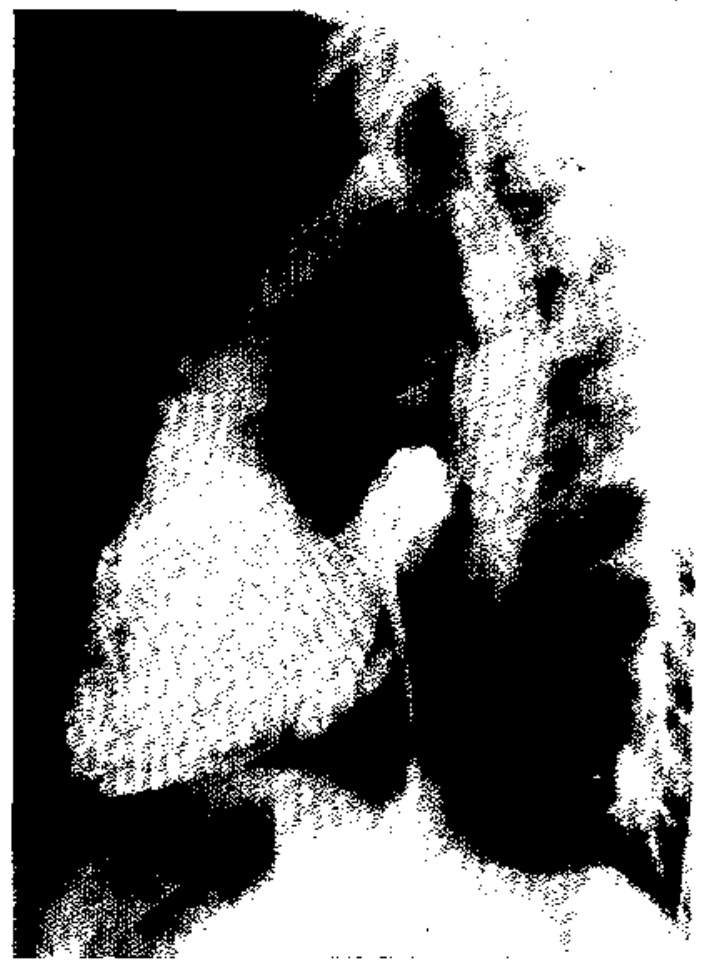

Fig. 4: Auriculografía icquierda en una transposición corregida aislada de los grandes vasos (proyección lateral izquierda). Olsérrese la morfología trabeculada del ventrículo arterial y la aorta anterior.

\section{DISCUSION}

Desde el punto de vista embriológico la TC se debe a un trastorno doble del desarrollo cardíaco; inversión del asa ventricular y detención de la rotación vectorial del bulbo, elemento que en definitiva determina la transposicion arterial $1-4$. Ambas anomalias son independientes una de la otra, quedando ello demostrado en los casos de inversión ventricular aislada documentos tanto en situs solitus como en situs inversus visce ralis.

E] hecho que un componente de la TC sea una transposición de los grandes vasos y el otro una anomalía adicional se refleja, por un lado, en que la transposición completa de los grandes vasos (TCGV) y la TC coinciden en varias características y por otro en ciertas particularidades de la TC que son atribuidas al elemento adicional, es decir a la inversión ventricular. Caracteres comunes son el predominio masculino y la misma proporción, tipo y frecuencia de las asociaciones; es así como en las TCGV la CIV y la estenosis pulmonar se encuentran en un 19 y $4 \%$ respectivamente, cifras similares a las descritas en la $\mathrm{TC}^{5,6}$. Sin embargo, la frecuencia de CIV con estenosis pulmonar es menor en la TCGV, siendo del orden de $13 \%$, en cambio en la TC se presenta en el $21 \% 5,6$. Esta combinación la encontramos en $42 \%$ de nuestros casos. La discrepancia puede deberse a que nuestros pacientes, a diferencia de otros autores, corresponden a pacientes vivos cuya sobrevida precisamente está favorecida por una estenosis pulmonar cuando hay CIV.

En resumen, la similitud en el predominio del sexo masculino y el paralelismo de las malformaciones asociadas dependen del trastomo bulbo truncal.

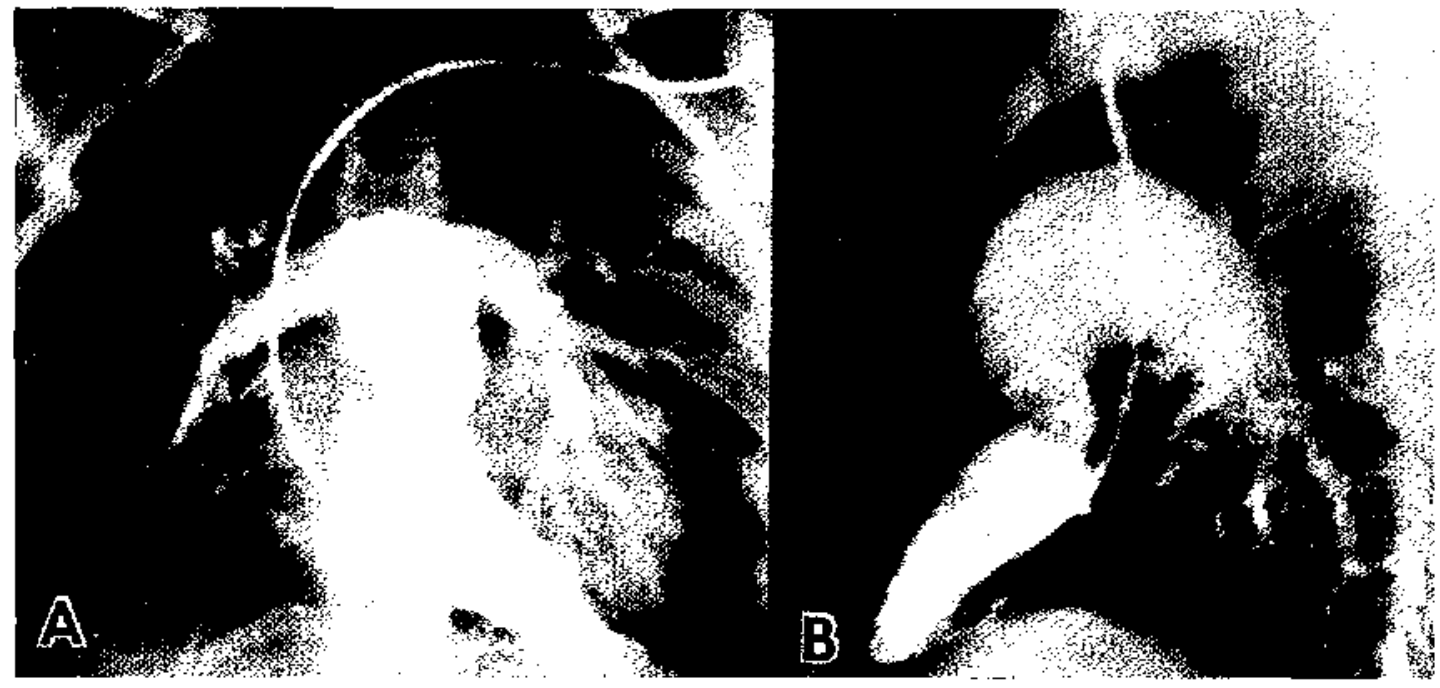

Fi. 3: Angiocardiagrafia sejectiva en ventrículo venoso en un caso de transposicrón corregida aislada de los grandes vasos: A) trontal, B) lateral izquierda. Obsérvese la arterja pulmonar medial y posterior y el ventrículo venoso de paredes lisas (morfologia de ventrículo izquierdo). 
Las particularidades de la $\mathrm{TC}$ se refieren, en cambio, a la elevada frecuencia de anomalías de la válvula atrioventricular izquierda y de bloqueos auriculoventriculares. La importancia de estas alteraciones radica en que del comportamiento de ellas depende básicamente el pronóstico de las TC.

La alteración de la válvula Iricúspide tiene una frecuencia que puede llegar al $90 \%$, describiendose disposición anatómica similar a la enfermedad de Ebstein en cerca de $70 \%$ de los cásos de $\mathrm{TC}^{5}, 6,7,8,9,10,11$. Otras malformaciones suelen ser diversos tipos de hipoplasias valvulares. El hecho que existá alteración de la configura. ción de los velos valvularcs no implica necesariamente compromiso hemodinámico. Se describe insuficiencia valvular tricuspidea en e] 30 a $50 \%$ de los casos con malformación valvular 1,2 , $7,9,12$. Se ha planteado como factor desencadenante de insuficiencia, el cierre quirúrgico de la CIV porque determina aumento de la presión cn el ventriculo izquierdo y ocasiona regurgitación tricuspídea en una válvulà anatómicamente predispuesta12,13. Sin embargo, se la estable. cido, como en el caso de los bloqueos a $v_{1}$ que la lesión de los velos aumenti progresivamente implicando el establecimiento de la insuliciencia y la consecuente progresión de ésta, en pacientes no intervenidos quirúrgicamente de CIV 12.

El bloqueo a--v, en sus diversas formas es una complicación frecuente de lia TC, oscilando entre 니 30 y $60 \%$ de los cesos $1.2,4,5,7,8,9,12$

Las alteraciones embriológicas del sistemal excitoconductor que explican los trastornos de conducción y la inversión de la actividad ventricular son: la inversión y elongación del haz de His. la discontinuidad de éste con el nódulo auriculoventricular y li duplicidad de este mismo tódulo 14, 15, 16. 17. En nuestra casuística encontramos un $12 \%$ de blogueos, en sus tres tipos; esta menor frecuencia en relación con to publicado nos parece atribuíble a la menor edad de rucstros pacienles yat que se ha establecido $y$ demostrado un aumento tanto de la incidencia como de la severidad de los bloqueas en la medida que so avanza en edads. La disposicion anatómica anterior del haz. de His determina que ocurra una elevada frccuencia de lesion del hac al realizarse la reparación quirárgica, específicamente de la CIV7. 12-17

La radiogralía simple de tórax aporla elementos propios originados por la malposición de los grandes vasos: debido a que la arteria pulmonar es medial, no forma parte del borde izquierdo de la silueta cardíaca. la que presenta sólo 2 arcos: el superior correspondiendo a aortil $y$ el inferior al ventrículo arteriat.

Los hallakgos hemodinámicos dependen de Jas anomalías asociadas. Se debe sospechar la TC cuando el paso hacia la arteria pulmonar es difícil o cuando al hacerlo la sonda adopta una posición medial y posterior. Es sin duda la angiografía selectiva la que permite el diagnóstico preciso ál demostrar la siluación anterior e izquicrda de la aorta, posterior $y$ derecha de la pulmonar, la morfología invertida de los ventrículos y las anomalías asociadas $1,2,18,19$. El signo de "la bayonetal" representado por la salida del tronco de la arteria pulnonar desde el ventriculo posterior siguiendo un trayecto ascendente, luego horizontal hacia atras, para alli bifurearse, resulta un elemento de ayuda diagnóstica.

La TC $\sin$ malformaciones asociadas es rara: con el tiempo cerca de la mitad de los casos llegan a presentar bloqueo a- $v$ y prácticamente todas desarrollan algún grado de insuficiencia tricuspidea 4, 7,8, 12, 21 . En nuestra casuistica encontramos sólo 2 casos de este tipo de TC, uno de ellos con sindrome de Wolff-Parkinson-White, y crisis de taquicardia paroxística.

La mortalidad derivada del tratamiento quirúrgito es elevada, entre 37 y $25 \%$. El pronóstico postoperatorio depende de la existencia de defectos residuales (CIV, estenosis pulmonar) de la función ventricular izquierda deterninada por el grado de compromiso funcional 1ricuspideo y e] grado de hipoxia y por último, del establecimiento de bloqueo a-v, en especial completo, después de la cirugial $12,13,15$.

Por las razones expuestas, la indicación de tratamiende quirúrgico on la TC dobe ser bien fundamentada. Se considera indicado cuando hay insuticiencia cardíaca de difícil manejo. cuando existe uл cortocircuito de derecha a izquietda (estenosís pulnonar más CIV) que esté determinato hipoxia considerable y cuondo exista una insuficiencia tricuspidea severa, que es la que generalmente hace inmanejable la insuficiencid cardiaca. A pesar que el objetivo de esta revisión no fue analizar al pronóstico ni resultados quirúrgicos, cabe ser̃alar que de los 10 pacientes operados en esta serie, 3 fallecicron $(30 \%$ ) durante la intervención.

El estudio clectrofisiológico intraperatorio del haz de Hiss, destinado básicamente para ubicar el recorrido del tejido cxcitoconductor ha demostrado ser beneficioso, disminuyendo en forma significátiva los bloqueos residuales.

\section{RESUMEN}

Se analizan las caracteristicas clínicas, hemodinámicas y angiocardiográficas de 43 casos de transposición corregida de los grandes vasos (TC). La edad promedio de los pacientes fue de 9,6 anos, con predoninio masculino (2:1). $38 \mathrm{TC}$ 
ocurrieron en situs solitus visceralis (31 con nomocardia, 7 dextroversiones) $y 5$ en situs inyersus visceralis (3 dextrocardias, 2 Ievoversiones).

Sólo 2 casos no tenían cardiopatía asociada (1 con un sindrome de Wolff-Parkinson-White).

La CIV fue la cardiopatía asociada más frecuente $(91 \%)$ ya sea aislada $(21 \%)$ o asociada a estenosis pulmonar (42\%) o a malformación de la válvula atrioventricular jzquierda (16\%) o a ésta y a estenosis pulmonar (12\%). La combinación de CIV y estenosis pulmonas es la asociación más frecuente $(42 \%)$, sigujendo en frecuencia la alteración de la válvula a-v izquierda $(30 \%)$ aistada o combinada.

Electrocardiográficamente, un alto porcentaje presentó inversión de la actividad eléctrica: ondas $Q$ en precordiales derechas, ausentes en izquierda en $70 \%$. Bloqueos a- $\vee$ de diversos grados en $12 \%$.

Radiológicamente, lo característico de la $\Gamma \mathrm{C}$ es un contorno anomal del borde jzqujerdo del corazón, el que está fomado sólo por 2 arcos (superior, aorta, inferior, ventrículo arterial).

Hemodinámicamente destaca la dificultad para entrar en arteria pulmonar y el trayecto anormal de la sonda al cateterizas los grandes vasos (arteria pulmonar medial y posterior, aorta, a la izquierda anterior). La angiocardiografía selectiva establece el diagnóstico de $\mathrm{TC}$ y de las cardiopatías asociadas.

Se analiza el efecto de Ja inversión ventricular sobre el sistema éxcito-conductor y sus consecuencias sobre lit producción de transtornos del ritmo, asj como los pobres resultados quirúrgicos de la corrección de las cardiopatias asociadas.

\section{REFERENCIAS}

1. Dupuis C. Kachaner J., Quero Jimenez M., Pernot C.. Rey C.: Cardiologie Pediatrique. Chap. 17. Flammarion Mcdicine-Sciences. Paris, Irancia.

2. Keith J., Rowe R., Vhad H.: Heart Disease in Infancy and Cluildhood. 3 th ed. New Yotk: Mc.Millan, 1978 .

3. Chuaqui $B$. Bersch $\boldsymbol{W}^{*}$.: The formal genesis of the transposition of the great arteries. Virchows Arch. Abst. Path. Anat. 358:11, 1973.

4. Von Pragh R.: What is congenitally corrected transposition? N. Fingl. J. Med. 282: 1097, 1970.
5. Schiebler G., Edwards J., Burchel H., Dushane J., Ongley P., Wood E.: Congenital corrected transposition of the great vassels: a study of 33 cases. Pediatrics 27: 851, 1961.

6. Anderson R., I.illehei W., Lester $R$.: Corrected transposition of the great vessels of the heart. A review of 17 cascs. Pediatrics 20:626, 1957.

7. Alluork $\mathcal{S}$, Bentall H., Becker A., Cameron $H$., Gerlis L., Witkinson $J$., Anderson R.: Congenjtally cotrected transposition of the great arteries: Morphologic study of the 32 cases. Am. J. Cardiol. 38: $910,1976$.

8. Shem-Tov A., Deutsh V., Yahini J., Krous $Y$., Nenfeld $H$.: Corrected tuansposition of the great arteries. A modified approach to the clinical diagnosis in 30 cases. Am. J. Cardjol. 27: 99, 1971.

9. Friedberg D., Nadas A.: Clinical profile of patients with congenital cortected transposition of the grcat arteries. N. Engl. J. Med. 282: 1053, 1970.

10. Pilapil V, Bennett $X$., Watson D.G., Lehon P.: Corrected tratosposition of the great arteries. Am. J. Med. 51: 482, 19?1.

11. Wotson $G$.: The diagnosis of corrected transposition of the great vessels. Brit. Heart. J. 26: 770, 1964.

12. Huang B., Browman F., Malm $I_{\text {., Krongrd }}$.: Surgical repair of congenitally corrected transpoxilion of the great arteries: results and follow-up. Am. J. Cardiol. 50: 781, 1982.

13. Metcalfe J., Somerville $J .:$ Surgical repair of lexions associated with corrected uransposition. Iate results. Brit. Heart. J. 50:476, 1983.

14. Kupersmith J., Krongrad E., Gersony W., Bowman $F .:$ rilectrophytiologic identification of the specialized conduction system in corrected trans. position of the great arteriex. Circulation 50:795, 1974.

15. Fox l., Kirklin J., Waldo A., Pacifico A, Bargeron L.: Intracardiac repair of cardiac malformations with atrioventricular discordance. Circulation 54: 123, 1976.

16. Waldo A., Pacifion A., Bargeron L., James $T$., Kirklin $J$ : liectrophysiological delineation of the specialized A-V conduction system in patients with cotrected transposition of the great vessels and ventricular septal defect. Circulation 52: 435, 1975.

17. Wilkinson $I$. Smith A., lincoln C., Anderson R.: Conduction tissues in congenitally corrected transposition with situs inversus. Brit. Heart J. 40: 41, 1978 .

18. Attic F., Soni J.. Orseyesitz J., Muñoz Castellanos L., Testelli $M .$, Buendia $A$. .: Angiographic studies of attioventricular discondance, Circulation 62: 407 , 1980.

19. Granja M., Sadi R., Gallo A., Kreutzer E.: Transposición corregiała de los grandes vasos. Análisis angiocardiográfico sccuencial en 36 pacientes. Rev Lat, Cardiol. Inf. ] : 189, 1985.

20. Rotem C.E.: Corrected transposition of the great vessels without associated defects. Am. Heart J. 70 : 305,1965 . 